\title{
Exploiting Dynamic Thermal Energy Harvesting for Reusing in Smartphone with Mobile Applications
}

\author{
Yuting Dai \\ College of Computer Science \& \\ Technology, Guizhou University \\ yutingdai90@gmail.com
}

\author{
Tao Li \\ IDEAL Lab, ECE Department, \\ University of Florida \\ taoli@ece.ufl.edu
}

\author{
Benyong Liu* \\ College of Big Data \& Information \\ Engineering, Guizhou University \\ byliu667200@163.com
}

\author{
Mingcong Song \\ IDEAL Lab, ECE Department, \\ University of Florida \\ songmingcong@ufl.edu
}

\author{
Huixiang Chen \\ IDEAL Lab, ECE Department, \\ University of Florida \\ stanley.chen@ufl.edu
}

\begin{abstract}
Recently, mobile applications have gradually become performance and resource intensive, which result in a massive battery power drain and high surface temperature, and further degrade the user experience. Thus, high power consumption and surface over-heating have been considered as a severe challenge to smartphone design.

In this paper, we propose DTEHR, a mobile Dynamic Thermal Energy Harvesting Reusing framework to tackle this challenge. The approach is sustainable in that it generates energy using dynamic Thermoelectric Generators (TEGs). The generated energy not only powers Thermoelectric Coolers (TECs) for cooling down hot-spots but also recharges micro-supercapacitors (MSCs) for extended smartphone usage. To analyze thermal characteristics and evaluate DTEHR across real-world applications, we build MPPTAT (Multi-comPonent Power and Thermal Analysis Tool), a power and thermal analyzing tool for Android. The result shows that DTEHR reduces the temperature differences between hot areas and cold areas up to $15.4^{\circ} \mathrm{C}$ (internal) and $7^{\circ} \mathrm{C}$ (surface). With TEC-based hot-spots cooling, DTEHR reduces temperatures of the surface and internal hot-spots by an average of $8^{\circ} \mathrm{C}$ and $12.8^{\circ} \mathrm{C}$ respectively. With dynamic TEGs, DTEHR generates $2.7-15 \mathrm{~mW}$ power, more than hundreds of times of power that TECs need to cool down
\end{abstract}

\footnotetext{
${ }^{*}$ Corresponding author

Permission to make digital or hard copies of all or part of this work for personal or classroom use is granted without fee provided that copies are not made or distributed for profit or commercial advantage and that copies bear this notice and the full citation on the first page. Copyrights for components of this work owned by others than ACM must be honored. Abstracting with credit is permitted. To copy otherwise, or republish, to post on servers or to redistribute to lists, requires prior specific permission and/or a fee. Request permissions from Permissions@acm.org.

ASPLOS '18, March 24-28, 2018, Williamsburg, VA, USA

(c) 2018 Association for Computing Machinery.

ACM ISBN 978-1-4503-4911-6/18/03 ..\$15.00

DOI: https://doi.org/10.1145/3173162.3173188
}

hot-spots. Thus, extra-generated power can be stored into MSCs to prolong battery life.

Keywords Mobile thermal analysis, waste heat harvesting, thermoelectric-based cooling, micro-supercapacitors storage

\section{ACM Reference format:}

Yuting Dai, Tao Li, Benyong Liu, Mingcong Song, Huixiang Chen. 2018. Exploiting Dynamic Thermal Energy Harvesting for Reusing in Smartphone with Mobile Applications. In ASPLOS '18: 8 Architectural Support for Programming Languages and Operating Systems, March 24-28, 2018, Williamsburg, VA, USA. ACM, New York, NY, USA, 14 pages. https://doi.org/10.1145/3173162.3173188

\section{Introduction}

Performance- and resource-intensive applications are gradually becoming the trend of smartphone apps, resulting in considerable energy drain along with high surface temperature. For example, Pokémon Go, one of the most popular apps, consumes 15 percent of battery usage within 30 minutes of play [1,2]. This high power drain app with high heat dissipation makes users quickly feel hot within ten minutes [3]. As a result, thermal and energy management is a critical part of mobile device design.

Traditionally, thermal and energy management techniques (e.g., fetch toggling, frequency throttling, dynamic thread migration and dynamic voltage and frequency scaling) work at the expense of performance. Among them, dynamic voltage and frequency scaling (DVFS) is the most widely used method to control the chip temperature to avoid hot-spots [4-6]. DVFS throttles the $\mathrm{CPU}$ frequency to reduce the input power, thus decreases the generated heat and avoids the high temperature.

However, due to the rapid increase of performance demand, the performance-intensive apps, such as deeplearning based apps [7], require high power consumption to achieve an acceptable user experience. The high power consumption then heats up the internal temperature quickly and continuously. Because these apps require high 
CPU frequency for performance demand, the default thermal management cannot reduce the generated heat through frequency scaling. Thus, the default thermal management is not suitable for these performanceintensive apps. Currently, backup batteries [8], active cooling fans [9] and PCM-based cooling methods [10] are applied to smartphones trying to solve these problems. However, this kind of cooling and charging methods significantly affect the user experience due to the space limitation of the smartphone. Furthermore, active cooling requires more power, which further lowers energy efficiency of the mobile device. Therefore, existing methods are the sub-optimal solution for smartphones to reduce surface temperature and prolong battery life.

To address the thermal issue of smartphones, we first build MPPTAT (Multi-comPonent Power and Thermal Analysis Tool) [11], a fine-grained real-time power and thermal analyzing tool based on Android, to evaluate the temperature maps of smartphones across different popular applications. We then analyze the internal and surface temperature distributions with MPPTAT and obtain several significant observations, as follows:

First, hot-spots, which exceed the human tolerable threshold $\left(45^{\circ} \mathrm{C}\right)[12,13]$ and directly lower the user experience, appear on the smartphone surface while running camera-intensive applications. Second, the smartphone's internal temperature differences between the hot areas and the cold areas are typically tens of degrees, which lead to an average temperature difference of $6.7^{\circ} \mathrm{C}$ in the smartphone surface. Third, the chip temperature exceeds $70^{\circ} \mathrm{C}$, when running resourceintensive applications. This high chip temperature would affect the lifespan of the chip.

Based on the above observations, we propose a mobile Dynamic Thermal Energy Harvesting Reusing (DTEHR) framework that exploits (1) Thermoelectric Generators (TEGs) [14] for waste heat harvesting, (2) Thermoelectric Coolers (TECs) [15] for hot-spots cooling, and (3) microsupercapacitors (MSCs) [16] for energy storage. Different from conventional static TEGs, we include a dynamic TEGs module (3-dimensionally mounted between the rear case and chip metal) in our DTEHR framework. The dynamic TEGs can manipulate the direction of heat transfer flow. Specifically, the dynamic TEGs can not only transfer heat from chip to ambient air but also transfer the heat from hot areas (e.g. the CPU) to cold components (e.g. the battery). Because hot-spots only appear at the CPU and camera, we mount Thermoelectric Coolers (TECs) behind the camera and the CPU to cool hot-spots below the human tolerable threshold. Moreover, since the number of our dynamic TEG pairs is much more than that of the TEC pairs, the power needed for cooling is much less than generated power from TEGs. Thus, we leverage one MSCs battery to store the extra-generated energy.

We deploy our TEGs, TECs and MSCs battery in the existing air block of the smartphone. Therefore, our DTEHR does not need extra space for deployment.
Moreover, the additional weight is only $2 g$. Overall, the physical overhead of our DTEHR is negligible.

To evaluate our DTEHR framework, we integrate DTEHR within MPPTAT. The results show that DTEHR reduces temperatures of both the internal and the surface hot-spots and keeps temperatures of the internal and the surface below $70^{\circ} \mathrm{C}$ and $41^{\circ} \mathrm{C}$, respectively. Thus, DTEHR improves the user experience and avoids affecting the chip lifespan. The temperature differences between hot areas and cold areas are reduced up to $15.4^{\circ} \mathrm{C}$ (internal) and $7{ }^{\circ} \mathrm{C}$ (surface). Moreover, the overall smartphone temperatures are decreased by an average of $12.8^{\circ} \mathrm{C}$ (internal) and $8^{\circ} \mathrm{C}$ (surface) as well. In addition, the generated power from dynamic TEGs not only powers TECs for hot-spots cooling but also recharges the MSCs battery. We summarize our contributions as follows:

1. We build MPPTAT, a power and thermal analyzing tool for Android. Based on this tool, we conduct the thermal characterization of the smartphone and motivate the opportunity for waste heat harvesting and hot-spots cooling.

2. To the best of our knowledge, this is the first work to introduce dynamic TEGs to balance the temperature difference while generating power.

3. Our proposed DTEHR is enough to power TECs to cool down the hot-spots, which greatly improve the user experience.

4. We deploy an MSCs battery in DTEHR to store the extra-generated energy from dynamic TEGs, which extends the battery life.

The rest of this paper is organized as follows: Section 2 provides background on MSCs, TEGs, and TECs. Section 3 presents the benchmark scenarios and thermal analysis with MPPTAT. We discuss the design of DTEHR in Section 4 and provide the evaluation results in Section 5. Section 6 discusses related work, and Section 7 concludes our paper.

\section{Background}

In this Section, we briefly introduce some background information about Thermoelectric Generators (TEGs), Thermoelectric Coolers (TECs) and micro-supercapacitors (MSCs). Section 2.1 presents the conception of MSCs. Section 2.2 presents the principle and mathematical model of TEGs and TECs.

\subsection{Micro-supercapacitors}

Traditionally, supercapacitors (SCs) are electrochemical devices, which store electrical charges based on the electrochemical double layer effect [17,18]. Typical SCs have higher power density and longer life while exhibiting similar electrical properties with other batteries. SCs have been used to replace or complement batteries in various macro-scale applications.

Recently, rapidly increasing demand for the development of miniaturized electronic devices has led to the high potential of micro-supercapacitors (MSCs) 


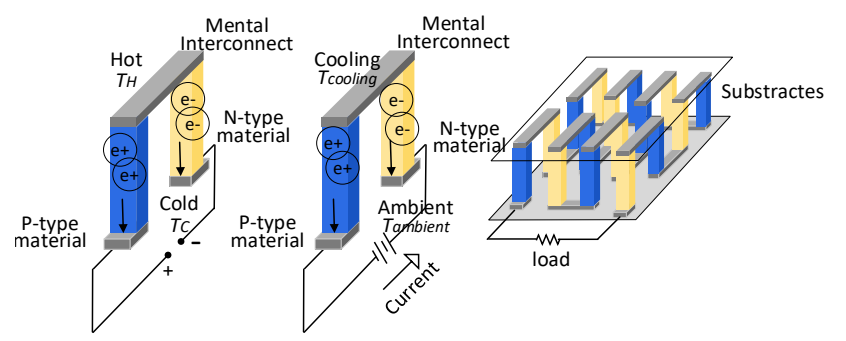

(a)

(b)

(c)

Figure 1 (a) Schematic of a TEG. (b) Schematic of a TEC. (c) Typical construction of TEGs or TECs.

[16][19-21]. The MSCs battery, fabricated by nanostructured materials with thin-film manufacture technology and device architecture, is an important onchip micro-scale energy storage for miniaturized devices.

\subsection{Thermoelectric Generators and Thermoelectric Coolers}

The thermoelectric effect, as the underlying working mechanism of TEGs and TECs, can directly create an open-circuit voltage from a temperature difference between two sides of thermoelectric transformation materials, and vice versa. When a temperature difference is subject to a thermoelectric transformation material, the heat transfer leads to an open-circuit voltage. Then a flow of electron current can be generated if the circuit is connected. This phenomenon, called the Seebeck effect, can be used as an electricity generator. Conversely, if a current flow through the material, the current then creates a temperature difference, called the Peltier effect, which can be used as a cooler.

One thermocouple (one TEG or TEC is shown in Fig. 1) means two electrically semiconductor materials: one $\mathrm{p}$ type (blue bar) and the other n-type (yellow bar) connected in series using metal interconnectors (grey bar). Fig. 1 (a) shows a schematic of a TEG pair. When a temperature difference occurs between two sides of the thermocouple, it absorbs heat from the hot side and releases the heat to the cold side. With the heat transfer, the positively charged electrons in the p-type material and the negatively charged electrons in the n-type flow from the interconnection to the base. Thus, a voltage difference between two base electrodes is generated. A large number of TEG pairs are connected in series to form a device as shown in Fig. 1 (c) to generate a larger voltage. When a current flows through the semiconductor as Fig. 1 (b) shows, the electrons in both types of semiconductor materials flow the same way as above, resulting in the cooling of the interconnection. TECs are also connected in series as shown in Fig. 1 (c) for quicker cooling.

As shown in Fig. 2, TEGs and TECs described above are inserted in an equivalent thermal circuit model, illustrating their thermal and electrical characteristics. In the case of Fig. 2 (a), $Q_{H}, Q_{C}$, and $Q_{\text {Power }}$ indicate the heat absorbed from the hot side, the heat released to the cold

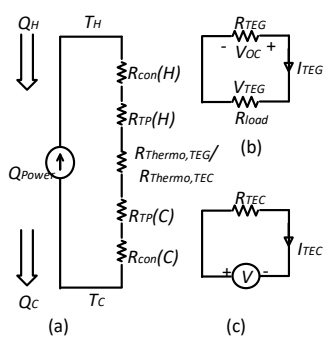

Figure 2 (a) Thermal circuit model of thermocouple elements. (b) Electric circuit model of TEGs. (c) Electric circuit model of TECs.

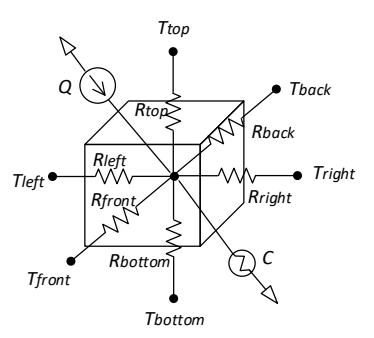

Figure 3 A simply part of the thermal RC network. side, and the energy generated from TEGs or received by TECs, respectively.

\subsubsection{Thermoelectric Generators}

To evaluate the amount of energy generated by TEGs, we first calculate the open-circuit voltage of the TEGs:

$$
V_{O C}=n \alpha_{T E G} \Delta T
$$

where $n$ is the number of TEG pairs; $\Delta T$ presents the temperature difference between the hot and cold interconnections of TEGs; and $\alpha_{T E G}$ is the Seebeck coefficient of TEGs, calculated as $\alpha_{T E G}=\alpha_{P}-\alpha_{N}\left(\alpha_{P}, \alpha_{N}\right.$ represent the Seebeck coefficients of the p-type and n-type semiconductors respectively).

With the external load resistance $\left(R_{\text {load }}\right)$, an electric current $\left(I_{T E G}\right)$ output from the TEGs in Fig. 2 (b) is calculated as:

$$
I_{T E G}=\frac{V_{O C}-V_{T E G}}{R_{T E G}}
$$

The generated current changes with varying TEGs output voltage $\left(V_{T E G}\right)$. In this circuit shown in Fig. 2 (b), the matching load point occurs when the output voltage equals the half of open-circuit voltage. In addition, the generated electric power of TEGs $\left(P_{T E G}\right)$ is calculated as:

$$
P_{T E G}=I_{T E G}^{2} R_{T E G}=\left(\frac{V_{O C}-V_{T E G}}{R_{T E G}}\right)^{2} R_{T E G}=\frac{\left(n \alpha_{T E G} \Delta T\right)^{2}}{4 R_{T E G}} \text { (3) }
$$

\subsubsection{Thermoelectric Coolers}

While applying a voltage to the TECs device, a current flows through the device. The current carries heat from the cooling side to the ambient side (as Fig. 1 (b) shows). According to Newton's law of heat transfer, the heat flux of TECs is shown as follows:

$$
Q_{K}=-k G \Delta T
$$

where $G$ is the geometrical factor of TECs which is equal to the cross-sectional area over the length of each TEC pair, and $k$ is the thermal conductivity of TEC elements.

According to the definition of Joule heat flux and Peltier effect, the Joule heat flux $\left(Q_{f}\right)$, the heat absorbed from cooling side ( $\left.Q_{\text {cooling }}\right)$ and the heat released to ambient side (Qambient) present as follows: 
Table 1 Description of chosen benchmark scenarios

\begin{tabular}{|c|c|c|c|}
\hline Type & Benchmark & Description & Operations on the App \\
\hline \multirow[b]{2}{*}{ Browsers } & Firefox & A widely used web browser. & $\begin{array}{l}\text { Launching the app, loading one pre-downloaded web } \\
\text { page, and scrolling over it at a pre-set speed. }\end{array}$ \\
\hline & Layar [27] & $\begin{array}{l}\text { It can scan publications and generates } \\
\text { multimedia information including videos, } \\
\text { graphs, etc. }\end{array}$ & $\begin{array}{l}\text { Launching the app, scanning the magazine } \\
\text { downloaded from the Layar website, and switching } \\
\text { pages every } 20 \text { seconds. }\end{array}$ \\
\hline \multirow{2}{*}{ Video Players } & MXplayer & A video playback application. & $\begin{array}{l}\text { Launching app, playing one chosen video for } 20 \\
\text { seconds, and pausing for } 1 \text { second after } 10 \text { seconds. }\end{array}$ \\
\hline & YouTube & An online video- sharing application. & $\begin{array}{l}\text { Launching app, playing one chosen video for } 20 \\
\text { seconds, and pausing for } 1 \text { second after } 10 \text { seconds. }\end{array}$ \\
\hline \multirow[t]{2}{*}{ Communication } & $\begin{array}{l}\text { Google } \\
\text { Hangout }\end{array}$ & $\begin{array}{l}\text { A communication application that includes } \\
\text { instant messaging, video chat, SMS and } \\
\text { VOIP features. }\end{array}$ & $\begin{array}{l}\text { Launching the app, sending a text message, and } \\
\text { initiating a } 30 \text {-second video call. }\end{array}$ \\
\hline & Facebook & $\begin{array}{l}\text { An online social media and social } \\
\text { networking application. }\end{array}$ & $\begin{array}{l}\text { Launching the app, scrolling feeds, opening up one } \\
\text { picture, and leaving a message. }\end{array}$ \\
\hline \multirow{3}{*}{ Games } & Quiver [28] & $\begin{array}{l}\text { A 3D MAR app that brings flat coloring } \\
\text { pages to 3D animation. }\end{array}$ & $\begin{array}{l}\text { Launching the app, clicking to start loading coloring } \\
\text { page, and capturing a } 20 \text {-second video from the } \\
\text { generated animation. }\end{array}$ \\
\hline & Ingress[29] & A location-based game. & $\begin{array}{l}\text { Launching the app, capturing 'portals' at a pre- } \\
\text { selected place, and linking them to create a virtual } \\
\text { triangle as a controlling symbol. }\end{array}$ \\
\hline & Angrybirds & $\begin{array}{l}\text { A puzzle video game, using a slingshot to } \\
\text { launch birds to kill all pigs within various } \\
\text { structures. }\end{array}$ & $\begin{array}{l}\text { Launching the app, entering the pre-selected stage, } \\
\text { and shooting two birds with one miss and one hit. }\end{array}$ \\
\hline \multirow{2}{*}{ Tools } & Blippar [30] & $\begin{array}{l}\text { A visual discovery app to unlock contents } \\
\text { users are curious about, which works like a } \\
\text { human eye. }\end{array}$ & $\begin{array}{l}\text { Launching the app, tapping the screen to identify } \\
\text { objects, and scanning prepared objects one by one. }\end{array}$ \\
\hline & $\begin{array}{l}\text { Google } \\
\text { Translate [31] }\end{array}$ & $\begin{array}{l}\text { One function of Google translate is to } \\
\text { translate the words scanned through the } \\
\text { camera. }\end{array}$ & $\begin{array}{l}\text { Launching the app and translating an academic paper } \\
\text { in AR mode. }\end{array}$ \\
\hline
\end{tabular}

$$
\begin{gathered}
Q_{J}=I_{T E C}^{2} R_{T E C} \\
Q_{\text {Cooling }}=\alpha_{T E C} I_{T E C} T_{\text {Cooling }} \\
Q_{\text {ambient }}=\alpha_{T E C} I_{T E C} T_{\text {ambient }}
\end{gathered}
$$

where $I_{T E C}$ is the assumed current applied to the TECs; $\alpha_{T E C}$ is the Seebeck coefficient of TECs, and $T_{\text {Cooling }}$ and $T_{\text {ambient }}$ represent the temperature at the cooling and ambient sides of the TECs respectively.

According to Fourier's Law [22], heat absorbed from the TECs (composed by $n$ TEC pairs) cooling side and released to the ambient side can be assumed as follows:

$$
\begin{aligned}
Q_{\text {Cooling }} & =2 n\left(Q_{\text {Cooling }}+Q_{K}-\frac{Q_{J}}{2}\right) \\
& =2 n\left(\alpha_{T E C} I_{T E C} T_{\text {Cooling }}-k G \Delta T-\frac{I_{T E C}^{2} R_{T E C}}{2}\right) \\
Q_{\text {ambient }} & =2 n\left(Q_{\text {ambient }}+Q_{K}+\frac{Q_{J}}{2}\right) \\
& =2 n\left(\alpha_{T E C} I_{T E C} T_{\text {ambient }}-k G \Delta T+\frac{I_{T E C}^{2} R_{T E C}}{2}\right)
\end{aligned}
$$

Combining with equations (8) and (9), the input electrical energy can be calculated as:

$$
\begin{aligned}
Q_{\text {Power }} & =Q_{\text {ambient }}-Q_{\text {Cooling }} \\
& =2 n\left[\alpha_{T E C} I_{T E C}\left(T_{\text {ambient }}-T_{\text {Cooling }}\right)+I_{T E C}^{2} R_{T E C}\right] \\
& =2 n\left(\alpha_{T E C} I_{T E C} \Delta T+I_{T E C}^{2} R_{T E C}\right)
\end{aligned}
$$

Equations (3) and (10) reflect the relationship between electric power and the temperature difference of the TEGs and TECs, which are used below to build our DTEHR framework.

\section{Thermal Characterization}

This Section provides the thermal characterization of mobile applications. We first present our power and thermal analyzing tool MPPTAT in Section 3.1 and then introduce our benchmark in Section 3.2. In Section 3.3, we analyze the thermal characterization across our benchmark, to motivate our study.

\subsection{MPPTAT}

MPPTAT is designed to analyze the power consumption and temperature distribution at the granular level of device hardware components, single app, and thread level. Based on the activity states of hardware components, we build our power model. We adopt an event-driven method to trace power related events from kernel and device drivers of each hardware component. We store all power related events in the buffer of Ftrace [23] using the trace_printk API. The information in Ftrace is from the lowest level close to the hardware. This obtained information ensures MPPTAT to acquire the power state changes within a minimum time delay, thus provides high power estimation accuracy. We then build our thermal model using compact thermal modeling (CTM) [24], a popular thermal behavior simulating technique that provides accurate thermal temperature estimation of critical points. To speed up the computation, we adopt Cholesky's decomposition method [25] in our MPPTAT, which receives the physical device model description file. Moreover, the outputs are simulated thermal maps of the 
Table 2 Hardware and Software Specifications

\begin{tabular}{|l|l|}
\hline CPU & $4 \times 2.0 \mathrm{GHz}$ Cortex-A53 $+4 \times 1.5 \mathrm{GHz}$ Cortex-A53 \\
\hline GPU & Mali-T628 Mp4 \\
\hline Memory & 3GB RAM \\
\hline Display & 5.2 inches, 1080 x 1920 pixels \\
\hline Android & Lollipop 5.0 .1 \\
\hline Linux Kernel & 3.10 .61 \\
\hline
\end{tabular}

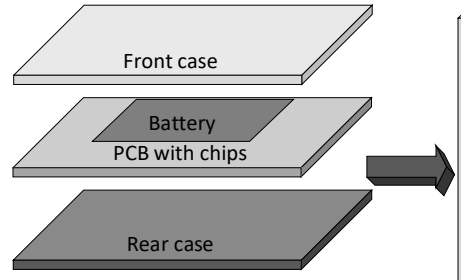

(a)

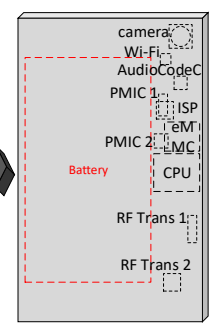

(b)
Figure 4 Schematic of smartphone.

device components, which is represented by one matrix each.

Fig. 3 shows a typical device component. After a time $\Delta t$, the temperature of the typical part is described as follows:

$$
\begin{aligned}
T^{\prime}= & T+\Delta T \\
= & T+\frac{P \Delta t}{C}+\frac{\Delta t}{C}\left(\frac{T_{\text {left }}}{R_{\text {left }}}+\frac{T_{\text {right }}}{R_{\text {right }}}+\frac{T_{\text {front }}}{R_{\text {front }}}+\frac{T_{\text {back }}}{R_{\text {back }}}+\frac{T_{\text {top }}}{R_{\text {top }}}+\frac{T_{\text {bottom }}}{R_{\text {bottom }}}\right)(11) \\
& -\frac{\Delta t}{C} \times T\left(\frac{1}{R_{\text {left }}}+\frac{1}{R_{\text {right }}}+\frac{1}{R_{\text {front }}}+\frac{1}{R_{\text {back }}}+\frac{1}{R_{\text {top }}}+\frac{1}{R_{\text {bottom }}}\right)
\end{aligned}
$$

where $P$ and $C$ are the electrical power consumption and the thermal capacitance of this component, respectively. $R_{\text {left }}, R_{\text {right }}, R_{\text {front }}, R_{\text {back }}, R_{\text {top }}$, and $R_{\text {bottom }}$ are the thermal resistances between this component and its left, right, front, back, top and bottom side components respectively; and $T_{\text {left }}, T_{\text {right }}, T_{\text {front }}, T_{\text {back }}, T_{\text {top }}, T_{\text {bottom }}$ represent the temperature of its surrounding components.

We use DAQ-USB-2408 [26] to validate our MPPTAT thermal model. Specifically, we measured three points on the smartphone (the CPU, the rear case under the CPU, and the midpoint of the screen) and then compare them with our simulation results. The test results show the error of our MPPTAT thermal model is less than $2^{\circ} \mathrm{C}$.

\subsection{Benchmark}

In this paper, 11 real-world Android applications (including browsers, video players, communication apps, games, social networks, and tools) are chosen based on popularity, with an emphasis on the emerging performance-intensive apps. We run each app based on real user behaviors, as described in Table 1. Each app is played for a fixed amount of time and repeated five times to avoid deviation.

\subsection{Thermal Analysis}

Table 2 lists the software and hardware specifications of our smartphone. Typically, the smartphone is composed

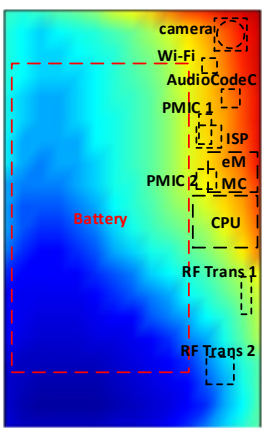

(a)

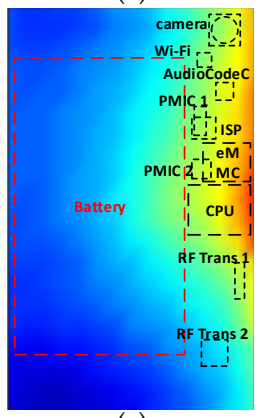

(c)

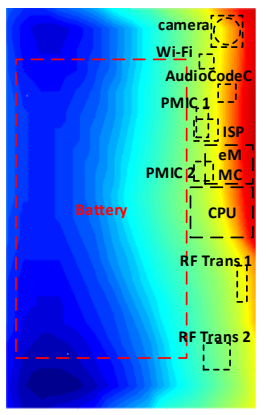

(e)

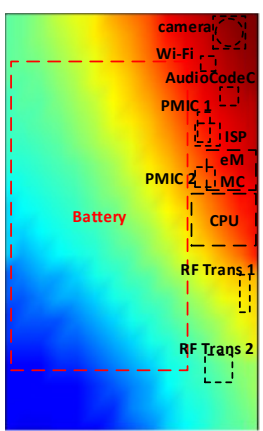

(b)

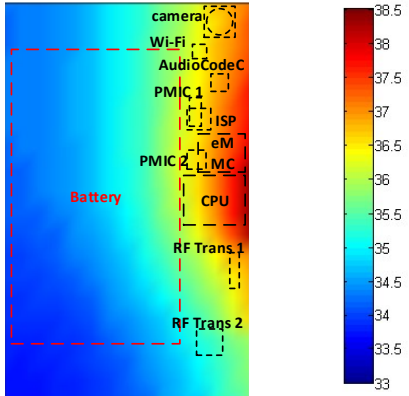

(d)

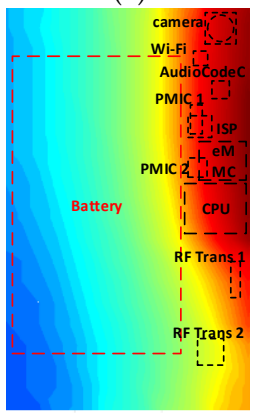

(f)
Figure 5 Temperature distributions on the both covers of smartphone (a) Front cover running Layar, (b) Back cover running Layar, (c) Front cover running Angrybirds, (d) Back cover running Angrybirds. (e) Front cover with cellular-only running Layar, (f) Back cover with cellular-only running Layar.

of different components: screen, camera, battery, CPU/GPU, DRAM, etc.. In other words, a smartphone can be divided into several layers by analyzing the spatial distribution of the individual components. Fig. 4 gives the simple schematic diagram of the smartphone in our analysis.

In Fig. 4 (a), we consider the screen protector and display as the first layer. In the second layer, chips are mounted on the PCB (Printed Circuit Board), which is located adjacent to the battery for reducing the thickness of the smartphone. The third layer is the rear case (the back plate) which acts as a battery holder. The significant components, mounted in the second layer, are shown in Fig. 4 (b).

The tests, measured by MPPTAT, are carried out to observe the temperature maps running various applications. The ambient temperature is $25^{\circ} \mathrm{C}$. We report 
Table 3 Overall temperature result obtained from smartphone

\begin{tabular}{|c|c|c|c|c|c|c|c|c|c|c|c|}
\hline & \multicolumn{2}{|c|}{ Browsers } & \multicolumn{2}{|c|}{ Video Player } & \multicolumn{2}{|c|}{ Social Media } & \multicolumn{3}{|c|}{ Games } & \multicolumn{2}{|c|}{ Tools } \\
\hline Applications & Layar & Firefox & MXplayer & YouTube & Hangout & Facebook & Quiver & Ingress & Angrybirds & Blippar & Translate \\
\hline \multicolumn{12}{|c|}{ Temperature of back cover surface } \\
\hline $\mathrm{T}_{\text {back, } \max }\left({ }^{\circ} \mathrm{C}\right)$ & 52.9 & 41.1 & 41.6 & 41.8 & 39.5 & 35.7 & 47.6 & 40.6 & 38.4 & 46.7 & 49.9 \\
\hline $\mathrm{T}_{\text {back, } \min \left({ }^{\circ} \mathrm{C}\right)}$ & 40.0 & 35.3 & 35.6 & 35.6 & 34.2 & 32 & 39.4 & 35.0 & 33.7 & 38.4 & 41.4 \\
\hline $\mathrm{T}_{\text {back,avg }\left({ }^{\circ} \mathrm{C}\right)}$ & 44.0 & 37.0 & 37.6 & 37.6 & 35.8 & 33.1 & 42.3 & 36.7 & 35.1 & 41.0 & 44.2 \\
\hline Spots areaback & $30.3 \%$ & $0 \%$ & $0 \%$ & $0 \%$ & $0 \%$ & $0 \%$ & $15 \%$ & $0 \%$ & $0 \%$ & $7 \%$ & $31.3 \%$ \\
\hline \multicolumn{12}{|c|}{ Temperature of internal components } \\
\hline $\mathrm{T}_{\text {internal, } \max \left({ }^{\circ} \mathrm{C}\right)}$ & 77.3 & 71.1 & 70.0 & 70.3 & 66.2 & 55.4 & 82.9 & 69.8 & 62.1 & 71.6 & 91.6 \\
\hline $\mathrm{T}_{\text {internal,min }}\left({ }^{\circ} \mathrm{C}\right)$ & 39.3 & 35.1 & 35.5 & 37.0 & 34.2 & 32.1 & 39.2 & 34.9 & 33.7 & 38.6 & 41.5 \\
\hline${\text { Tinternal,avg }\left({ }^{\circ} \mathrm{C}\right)}$ & 50.4 & 42.6 & 43 & 44.7 & 42.6 & 36.3 & 49.3 & 42.1 & 39.6 & 46.6 & 54.6 \\
\hline \multicolumn{12}{|c|}{ Temperature of front cover surface } \\
\hline $\mathrm{T}_{\text {front } \max \left({ }^{\circ} \mathrm{C}\right)}$ & 51.0 & 40.2 & 40.7 & 41.1 & 38.6 & 35.2 & 46.3 & 39.7 & 37.7 & 45.2 & 48.6 \\
\hline $\mathrm{T}_{\text {front } \min \left({ }^{\circ} \mathrm{C}\right)}$ & 38.8 & 34.7 & 35.1 & 35.8 & 33.6 & 31.7 & 38.7 & 34.5 & 33.3 & 37.8 & 40.6 \\
\hline $\mathrm{T}_{\text {front avg }\left({ }^{\circ} \mathrm{C}\right)}$ & 42.2 & 36.5 & 36.9 & 37.8 & 35.3 & 33.2 & 41.4 & 36.2 & 34.8 & 40.4 & 43.6 \\
\hline Spots areafront & $15 \%$ & $0 \%$ & $0 \%$ & $0 \%$ & $0 \%$ & $0 \%$ & $6 \%$ & $0 \%$ & $0 \%$ & $0.3 \%$ & $22.3 \%$ \\
\hline
\end{tabular}

temperatures of the surface (both back cover and front cover of the smartphone) and of the internal components in Table 3. Each column of Table 3 refers to the execution of one mobile application. Typically, human skin can tolerate temperature up to $45^{\circ} \mathrm{C}$ for sustained use $[12,13]$. Thus, we also show the percentages of the area that exceed the threshold of human tolerance (Spots area in Table 3).

We notice that both sides of smartphone surfaces have a similar temperature distribution. Figs. 5 (a) to (d) show the temperature maps on both front and back surfaces of the smartphone while running Layar and Angrybirds with Wi-Fi. It is evident that front and back covers have a similar surface temperature distribution. The high power consumption of internal components (such as the CPU, camera, and Wi-Fi) not only heat-up themselves but also dissipate the heat to their surrounding areas. The temperature of both front and back surfaces increase from the heat dissipation of the internal layer. As the internal chips are mounted closer to the back cover, the average temperature of the back surface is a little higher than that of the front.

Figs. 5 (e) and (f) show the temperature maps of both front and back surfaces with cellular-only while running Layar. The temperature distribution with cellular-only is slightly different from that with $\mathrm{Wi}-\mathrm{Fi}$. While using the smartphone with cellular-only, the surface of $R T$ Transceiver 1 and 2 show an average higher temperature of $4^{\circ} \mathrm{C}$ than that running with Wi-Fi. Cellular consumes around $0.1 \mathrm{~W}$ more power than that running with $\mathrm{Wi}-\mathrm{Fi}$, resulting in a higher temperature at RT-Transceiver. Though RT-Transceiver show a higher temperature with cellular-only, the hot-spots occur the same place (the CPU and the camera) as that with Wi-Fi. Moreover, the average temperature with cellular-only is almost same as that with Wi-Fi.

While running camera-intensive apps, the surface temperature of hot-spots exceeds human tolerance. The existing thermal governor, dynamic voltage and frequency scaling (DVFS), changes CPU frequency to control the chip temperature. It throttles the CPU frequency to avoid hot spots and keeps the temperature below $45^{\circ} \mathrm{C}$. However, camera-intensive apps (Layar, Quiver, Blippar, and Google Translate) continually occupy the camera and other system resources, which result in the quick and continuous heat-up in the internal components. Because these apps require high CPU frequency for performance demand, the existing thermal governor is not suitable for these apps. The maximum surface temperature of cameraintensive apps exceed $45^{\circ} \mathrm{C}$, and among all chosen apps, Google Translate with AR mode shows the maximum percentage of the Spots areas $(31.3 \%$ on the back cover and $22.3 \%$ on the front cover).

One more observation about surface temperature distribution is that the temperature differences between the hot and cold areas are as much as $12.9^{\circ} \mathrm{C}$ and $12.2^{\circ} \mathrm{C}$ in the back and front surfaces respectively. The sizeable temperature difference directly affects the user experience, which is a severe challenge for the future design of smartphones.

We also show the summary of significant internal components' temperature in Table 3. The maximum internal temperature all exceeds $70^{\circ} \mathrm{C}$ while running resource-intensive applications, such as video processing apps (MXplayer and YouTube), apps with frequent use of Wi-Fi (Layar, Firefox, and Blippar) and camera-intensive apps (Quiver and Google Translate, etc.). The high temperatures caused by such apps consume more power and result in higher heat generation. Moreover, the temperature differences between different internal components are typically tens of degrees. The internal temperature differences between significant components range from $23.3^{\circ} \mathrm{C}$ (Facebook) to $50.1^{\circ} \mathrm{C}$ (Google Translate) and show an average difference of $35.2^{\circ} \mathrm{C}$. The large internal temperature difference gives an opportunity for us to design the dynamic TEGs for waste heat harvesting.

\section{Design of DTEHR}

In this Section, we discuss DTEHR framework in both dynamic TEGs and the generated energy reusing in detail. We first present the layout of TEGs, TECs and MSCs on the baseline architecture and then discuss the design for 


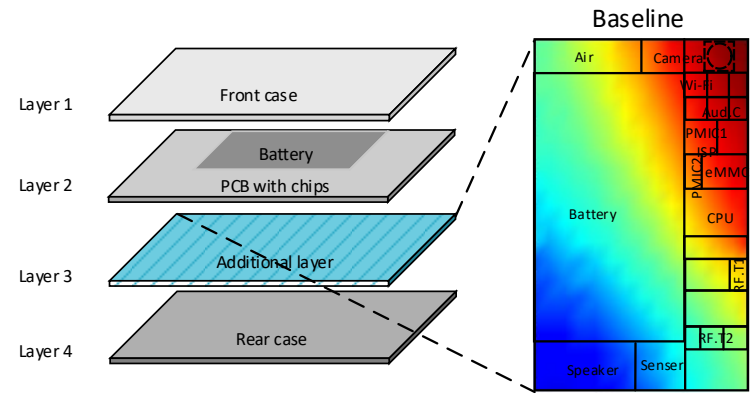

(a) (b)

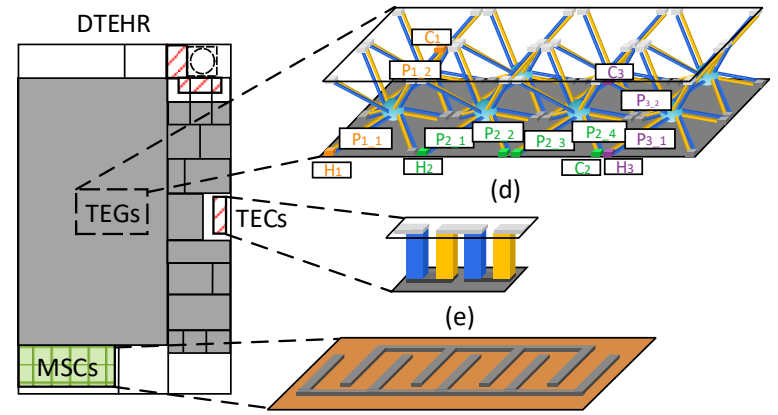

(c)

(f)

Figure 6 Self-cooling architecture (a) additional layer sits in between rear case and PCB, (b) temperature map of the additional layer, (c) the placement of TEGs, TECs and MSCs, (d) structure of the dynamic TEGs module, (e) structure of the TECs module, (f) structure of the MSCs module.

power-generating and energy-reusing, respectively. Finally, we introduce the operating modes in DTEHR.

\subsection{The Layout of DTEHR}

In our DTEHR framework, we use dynamic TEGs to harvest smartphone waste heat from the temperature differences between the internal components. The generated power can be used for two purposes. One is the cooling power (sustaining the power requirement of hotspots cooling by TECs) and the other is recharging power (recharging the MSCs battery to prolong the battery life). Fig. 6 (a) depicts the layout of the DTEHR framework. An additional thermoelectric material layer is applied under PCB board and battery, replacing half of the original air block.

Fig. 6 (b) shows the temperature map of the additional layer with an ambient temperature of $25^{\circ} \mathrm{C}$ while running Layar. The temperature difference between different components goes up to $38^{\circ} \mathrm{C}$. The temperature of the hot areas (near the $\mathrm{CPU}$, camera, and $\mathrm{Wi}-\mathrm{Fi}$ ) are all higher than $75^{\circ} \mathrm{C}$, whereas that of the cold spots (behind the battery and speaker) are all lower than $40^{\circ} \mathrm{C}$.

Based on this thermal distributional characterization, we choose the locations of TEGs and TECs modules and MSCs battery in DTEHR. Fig. 6 (c) illustrates the placement of TEGs and TECs, which consume $7000 \mathrm{~mm}^{2}$ and $50 \mathrm{~mm}^{2}$, respectively. The diagonal-striped blocks represent the functional units related with TECs, grey blocks represent units related with TEGs, square-striped blocks represent the placement of the MSCs battery, and the white blocks are left for the connection between TEGs and TECs. As discussed in Section 3.3, the hot-spots occur at the $\mathrm{CPU}$ and the camera (while running cameraintensive apps). Therefore, these two components are considered as our cooling targets and mounted with TECs for active cooling. On the other hand, Wi-Fi, eMMC, AudioCODEC, PMIC, ISP, RF Transceiver, and battery are placed with TEGs for generating power due to their lower temperatures.

\subsection{Power-Generating using TEGs}

The grey blocks in Fig. 6 (c) represent the units mounted with TEGs. [32-34], and [6] have shown that the temperature of each component in the smartphone only increases rapidly in the first tens of seconds after the current is fluxed through the component. After that, the temperature shows little change, and the internal temperature distribution almost keeps steady. Based on this conclusion, we assume that the temperature distribution of both sides of the additional layer keeps constant. When an application is started, the internal temperature of smartphone keeps constant after several seconds; it is possible that TEGs generate a stable power until usage changes (e.g., killing the app or opening another app).

Based on the significant internal temperature difference, DTEHR determines the dynamic TEGs module. Fig. 6 (d) shows the detailed structure of the proposed dynamic TEGs. The yellow and blue cubes represent the n-type and p-type TEG tiles, respectively. The balls represent the switches that control the connection of TEGs. The top and bottom substrates, mounted with metal wire, connect to Layer 2 and Layer 4 in Fig. 6 (a), respectively. Figs. 7 (a) and (b) illustrate the top view of two layers of the TEGs block in Fig. 6 (d).

Our proposed DTEHR has more freedom for TEG pairs. Different from conventional TEGs (shown in Fig. 1 (c)), our proposed dynamic TEGs could change their hot and cold connection points and paths. Eight thermal acquisition point (four at the top substrate and four at the bottom substrate) constitute one TEG block. The switches are mounted at each point and the center of the block. Fig. 7 (c) shows the detail of how switches are connected. $\mathrm{H}_{1}$, $\mathrm{H}_{2}$, and $\mathrm{H}_{3}$ are the hot sides of different TEG pairs; $\mathrm{C}_{1}, \mathrm{C}_{2}$, $\mathrm{C}_{3}$, and $\mathrm{C}_{4}$ are the cold sides of different TEG pairs. In other words, $\left(\mathrm{H}_{1}, \mathrm{C}_{1}\right),\left(\mathrm{H}_{2}, \mathrm{C}_{2}\right),\left(\mathrm{H}_{3}, \mathrm{C}_{3}\right)$ are three TEG pairs. $\left(\mathrm{P}_{1 \_1}, \mathrm{P}_{1 \_}\right),\left(\mathrm{P}_{2 \_1}, \mathrm{P}_{2 \_}, \mathrm{P}_{2 \_3}, \mathrm{P}_{2 \_}\right)$, and $\left(\mathrm{P}_{3 \_1}, \mathrm{P}_{3 \_2}\right)$ are their internal paths, respectively. At the hot side, the $\mathrm{n}-$ and $\mathrm{p}$ type tiles connect each other; and at the cold side, tiles 


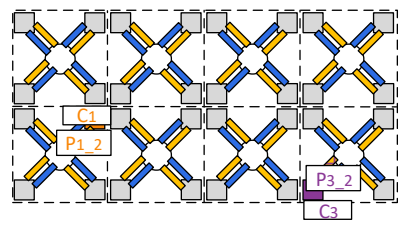

(a)

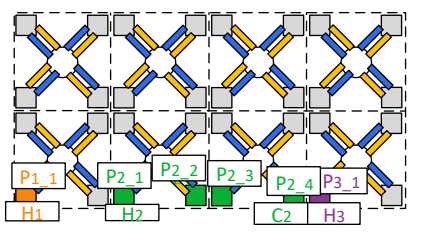

(b)

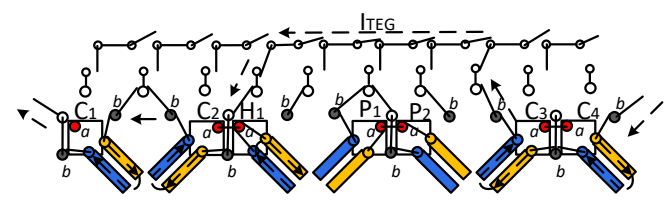

(c)

Figure 7 (a) top view of top layer of the dynamic TEGs block, (b) top view of bottom layer of the dynamic TEGs block, (c) different switch connection modes of dynamic TEGs.

connect to the other type tile of their neighbor TEGs; at the internal paths, tiles connect to the same type tiles. The different connection modes of switches are shown below:

Mode 1: At the hot side of one TEG pair (e.g., $\mathrm{H}_{1}$ in Fig. 7 (c)), the switches of $\mathrm{n}$ - and p-type tiles all turn to terminal 'a' so that the $\mathrm{n}$ - and $\mathrm{p}$-type tiles connect to each other.

Mode 2: At the cold side of one TEG pair (e.g., $\mathrm{C}_{1}, \mathrm{C}_{2}$, $\mathrm{C}_{3}, \mathrm{C}_{4}$ in Fig. 7 (c)), the $\mathrm{n}$ - and p-type tiles connect to other TEG pairs in series. The switches of both $n$ - and p-type tiles turn to terminal ' $b$ '. Thus, the n-type tile can connect with the p-type tile of the neighbor TEG pair, and the $\mathrm{p}$ type tile can connect with the n-type tile of another neighbor TEG pair.

Mode 3: At the internal path of one TEG pair (e.g., $\mathrm{P}_{1}$ and $\mathrm{P}_{2}$ in Fig. 7 (c)), the switch of the p-type tile turns to terminal ' $b$ ', while the switch of the n-type tile turns to terminal ' $a$ '. Thus, the p-type tile connects with the next p-type tile, and so is the n-type tile, resulting in a longer path of TEG pair.

According to the above modes, every TEG pair absorbs heat from the hot side and transfers to the cold side through their paths. The main idea of our method is to switch the operating modes to find the optimal trade-off between generated power and increasing temperature of the cold components. The algorithm is equipped with the following optimization:

$$
\begin{aligned}
& \text { Objective: } P_{T E G}= \max \Sigma_{i=1}^{N_{T E G}} \frac{\alpha_{T E G} \Delta T_{i}}{4 R_{i}} \\
& \text { Constrains: }:\left\{\begin{array}{l}
\Delta T_{i}>10^{\circ} \mathrm{C} \\
T_{i_{\text {hot }}}>T_{i_{\text {cold }}} \\
i \in\left[1: N_{T E G}\right]
\end{array}\right.
\end{aligned}
$$

where $P_{T E G}$ is the power generated from TEGs; $N_{T E G}$ is the number of TEG pairs; $\alpha_{T E G}$ is the Seebeck coefficient of TEGs; $\Delta T_{i}$ and $R_{i}$ are the temperature difference and electrical conductivity of $i_{t h}$ TEG pair, respectively; $T_{i_{h o t}}$ and $T_{i_{\text {cold }}}$ are temperatures of the hot and cold sides of $i_{t h}$ TEG pair. When the temperature difference is less than $10^{\circ} \mathrm{C}$, the generated power decreases to a low level that is not worth performing the dynamic computation. Therefore, we set the minimum threshold of temperature difference as $10^{\circ} \mathrm{C}$.

Our dynamic TEGs module generates energy caused by the temperature difference between different components or components with the ambient air. It can manipulate the direction of heat transfer flow. In other words, the dynamic TEGs can not only transfer heat from chip up to ambient air but also transfer the heat from hot areas (e.g. the CPU) to cold components (e.g. the battery). Thus, they can significantly reduce the temperature difference.

\subsection{Energy Reusing for TECs and MSCs}

Although our dynamic TEGs can reduce the temperature difference among different components, temperatures of hot-spots still increases with the substantial power usage while running performance-intensive applications.

To tackle this issue, we use TECs as an active cooling method to cool down hot-spots. By cooling hot-spots selectively, a smartphone can be used with powerintensive apps for a long time without 'burning' the surface, leading to a reduction in internal temperature without affecting chip lifespan. Since hot-spots appear around the camera and the CPU, TEC pairs are placed behind the camera and the CPU (shown in Fig. 6 (e)). These TEC pairs are designed in two modes, as follows:

Mode 1: (Power generating mode) TECs are connected with TEGs in series to generate power while running low power consumption apps or common usages such as calling or messaging.

Mode 2: (Spot cooling mode) When the hot-spots temperature exceeds $65^{\circ} \mathrm{C}$ (Thope, the threshold of activating the TECs module), TECs disconnect with TEGs and a flow of current applied to TECs to power them for spot cooling.

In spot cooling mode, electrical energy is used to power TECs to cool down the hot-spots. The reduced temperature is proportional to the input power. The optimization goal of TECs is to find the trade-off between the input power and decreasing temperature of the hotspots. The objective is derived as:

$$
\begin{gathered}
\text { Objective: } \min P_{T E C} \\
=\sum_{i=1}^{N_{T E C}} 2\left(\alpha_{T E C} I_{i}\left(T_{\text {ambient }}(i)-T_{\text {Cooling }}(i)\right)+I_{i}^{2} R_{i}\right) \\
\text { Constrains: }\left\{\begin{array}{c}
P_{T E C} \leq P_{T E G} \\
T_{\text {hope }} \leq T_{\text {Cooling }}(i)<T_{\text {die }} \\
T_{\text {ambient }}<45^{\circ} \mathrm{C} \\
i \in\left[1: N_{T E C}\right]
\end{array}\right.
\end{gathered}
$$

where $P_{T E C}$ is the power TECs required for hot-spots cooling; $N_{T E C}$ is the number of TEC pairs; $\alpha_{T E C}$ is the Seebeck coefficient of TECs. $I_{i}$ and $R_{i}$ are the input current and electrical conductivity of the $i_{t h}$ TEC pair, 


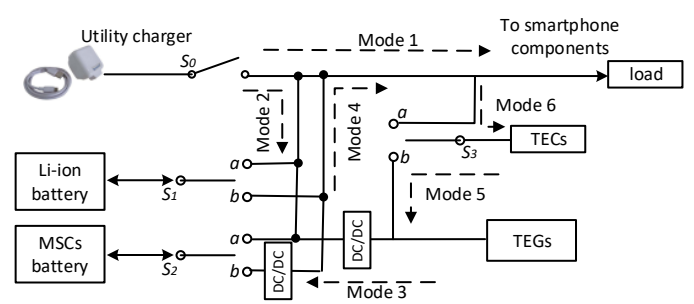

Figure 8 The hardware structure of DTEHR.

respectively; $T_{\text {ambient }}(i)$ and $T_{\text {Cooling }}(i)$ are temperatures of the ambient and cooling sides of the $i_{t h}$ TEC pair.

We use the power generated from TEGs to power the TECs for hot-spots cooling. The power supply for TECs should be lower than the generated power provided by TEGs. TECs absorb heat from internal chips and release it to the ambient air at the hot-spots. The surface temperature, which is directly affected by the heat released from TECs, should be kept under the threshold of the tolerable temperature of human $\left(45^{\circ} \mathrm{C}\right)$. When the internal temperature exceeds $65^{\circ} \mathrm{C}$, the temperature of the surface would reach the human tolerable limit. Therefore, we set the threshold to power TECs as $65^{\circ} \mathrm{C}\left(T_{\text {hope }}\right) . T_{\text {die }}$ is the temperature threshold that may cause dielectric breakdown. The cooling side temperature should remain below $T_{\text {die }}$ to avoid phone crashes.

In conclusion, TECs first stay in Mode 1. Then while running power-intensive apps, the power consumption jumps up to a high level and temperatures of the camera, and the CPU then quickly increase, making temperatures of the internal hot-spots exceed $T_{\text {hope }}$. At this time, TECs turn into Mode 2 to cool down the internal temperature of hot-spots and keep the surface temperature under $45^{\circ} \mathrm{C}$. The reduced temperature of hot-spots is monitored during runtime. When the hotspots' temperature is under the temperature of other units placed with TEGs, TECs turn into Mode 1.

As shown in Fig. 6 (c), the units of TEGs is hundreds of times larger than that of TECs. Besides, DTEHR only powers TECs for hot-spots cooling when the temperature exceeds $T_{\text {hope }}$. Thus, the power needed for TEC-based cooling is much lower than the power generated from TEG-based energy harvesting. Then the extra-generated power is stored in the MSCs battery for extended usage of the smartphone. We choose MSCs battery instead of traditional tiny coin battery because of its high power density and longer life. The high recharging frequency in DTEHR challenges the traditional battery's lifetime.

In this work, the MSCs battery is used as the energy storage for DTEHR for continuous waste heat harvesting. As the mobile phone is usually designed with an input voltage of $3.7 \mathrm{~V}$, the MSCs battery is connected to two $\mathrm{DC} / \mathrm{DC}$ converters. One DC/DC converter serves as a charger to the MSCs from the TEGs. The other DC/DC converter is used to match MSCs voltage with the mobile phone requirement of $3.7 \mathrm{~V}$.

\subsection{Operating Modes of DTEHR}

Fig. 8 illustrates the hardware structure of our DTEHR framework, which consists of one thermoelectric charger (power generated from TEGs), one utility charger, one TEGs module, one TECs module, one Lithium-ion battery, one MSCs battery with two DC/DC converters and several relays (S0-S3). In this subsection, we primarily introduce the six operating modes in DTEHR controlled by four relays $(\mathrm{S} 0-\mathrm{S} 3)$ :

Mode 1: When the bypass switch S0 turns on, the smartphone is in a normal charging mode. The conventional utility power is used to charge the battery and support smartphone's usage.

Mode 2: In this mode, the bypass switch S0 turns on, and S1 connects to terminal 'a'. The conventional utility power is used to charge the Lithium-ion battery as existing smartphones.

Mode 3: In this case, relay S2 connects to terminal ' $a$ '. The smartphone charged the MSCs battery using TEGs, which leads to an extended battery life before recharging.

Mode 4: When one battery is used to supply power to the smartphone, relay S1 or S2 (which relates to the power supplying battery) switches to terminal ' $\mathrm{b}$ '. That battery supports smartphone's use until it is empty.

Mode 5: In this mode, in which temperatures of the internal hot-spots stays under our given threshold ( $T_{\text {hope }}$ ), the TECs stay in power generating mode. Relay S3 switches to terminal 'b', the TECs module connects with the TEGs module in series for power generation.

Mode 6: When temperatures of the internal hot-spots exceed $T_{\text {hope }}$, relay S3 is switched to terminal 'a' to power the TECs for cooling the hot-spots. TECs module continues to cool down hot-spots until the spots' temperatures under temperatures of other TEGs mounted units or below our given threshold.

The above operating modes can be combined depending on the power demand and temperatures of the internal hot-spots (the CPU and the camera). We propose a power management policy to determine the working combination of these operating modes.

When the smartphone connects to the USB cable, the priority is to estimate the power demand of the smartphone. If the utility cannot meet the demand, and batteries are not empty, DTEHR works in Mode 1 (smartphone powered by the utility), Mode 4 (smartphone powered by batteries), while the MSCs battery keeps charging (Mode 3) until it is full. Apart from this, the utility is the only power supply for the smartphone (Mode 1). At this time, Lithium-ion battery is charged using USB cable (Mode 2) and MSCs battery is charged from TEGs (Mode 3) until they are fully charged.

When no utility power supplied or USB is disconnected from the smartphone, batteries are the only power supplement (Mode 4). The MSCs battery keeps charging (Mode 3) until it is full or the Lithium-ion battery is empty. 
Table 4 Physical parameters of TEGs and TECs modules

\begin{tabular}{ccc}
\hline & TEGs & TECs \\
\hline Thermal Conductivity $(\mathrm{W} / \mathrm{m} \times \mathrm{K})$ & 1.5 & 17 \\
Electrical Conductivity $(\mathrm{S} / \mathrm{m})$ & $1.22 \times 10^{5}$ & 925.93 \\
Specific Heat $(\mathrm{f} / \mathrm{kg} \times \mathrm{K})$ & 544.28 & 162.5 \\
Seebeck Coefficient $(\mu \mathrm{V} / \mathrm{K})$ & 432.11 & 301 \\
Density $\left(\mathrm{kg} / \mathrm{m}^{3}\right)$ & 7528.6 & 7100 \\
\hline
\end{tabular}

Moreover, TECs turn to spot cooling mode (Mode 6) when the internal hot-spots temperature exceeds $T_{\text {hope }}$. Otherwise, it works in Mode 5.

\section{Evaluation Results}

In this Section, we present the evaluation results of DTEHR by comparing it with two baseline strategies: statically TEG-based hot-spots cooling (baseline 1) and non-active cooling (baseline 2).

Statically TEG-based hot-spots cooling exploits only static TEGs (the stationary TEGs structure like Fig. 1(c)), which is fixed in the additional layer. The static TEGs transfer heat from the chip to ambient air to generate electrical energy. TEC-based hot-spots cooling (structured like Fig. 6(e)) placed the same as DTEHR. Non-active cooling is an ordinary smartphone without any active cooling method, utilizing DVFS as the only cooling method to avoid hot-spots.

\subsection{DTEHR Simulation Setup}

We integrate DTEHR model into MPPTAT and add the additional information (including position, size and material properties of TEGs, TECs, and MSCs) into the input description file. Thus, the updated description file and MPPTAT simulated power trace act as the input of DTEHR thermal model. Then the generated power of TEGs, power consumption of TECs as well as power input of MSCs is calculated to update the power trace for the thermal result of DTEHR.

We simulate an additional layer hosting one MSCs module, 6 TEC pairs and 704 pairs TEG tiles. To model the effects of TEGs and TECs, we first compute the temperature map according to the power consumption of different components. The second step is calculating the generated power from TEGs as well as that supplied to TECs and MSCs. We run a background process on the smartphone to perform the calculation. Since we only need to calculate the power generated between one point and its neighboring points, the additional power consumption of this process is negligible. After that, the calculated power is added to the TEGs, TECs and MSCs modules, and updating the temperature in the first step until the calculated power converges.

MSCs have a power density of $200 \mathrm{~W} / \mathrm{cm}^{3}$. The physical parameters of TEGs and TECs, shown in Table 4, are used in our simulation experiments. The TEGs and TECs modules are based on $\mathrm{Bi}_{2} \mathrm{Te}_{3}$ compounds [35,36] and $\mathrm{Bi}_{2} \mathrm{Te}_{3} / \mathrm{Sb}_{2} \mathrm{Te}_{3}[37,38]$ compounds, respectively. The

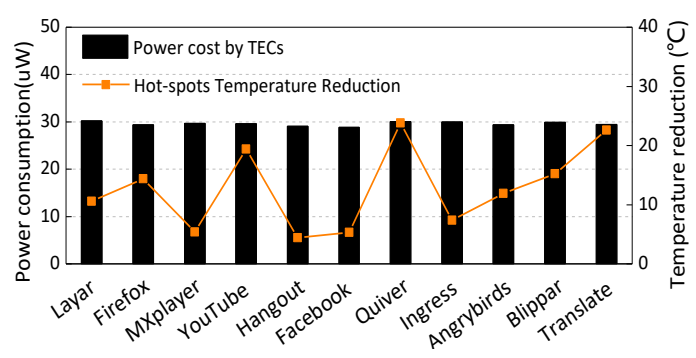

Figure 9 Cooling power consumption of the TECs and the corresponding hot-spots temperature reduction under DTEHR.

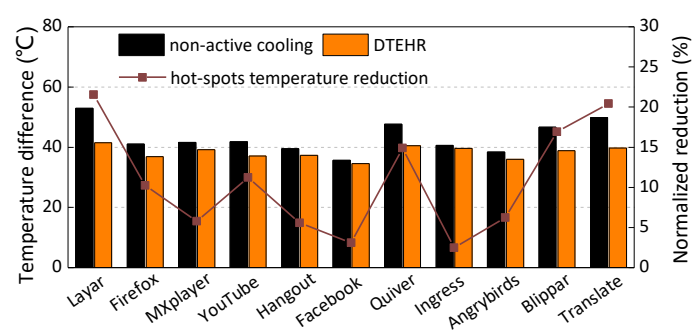

(a)

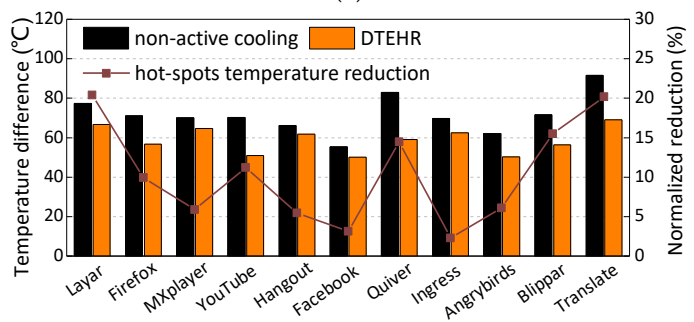

(b)

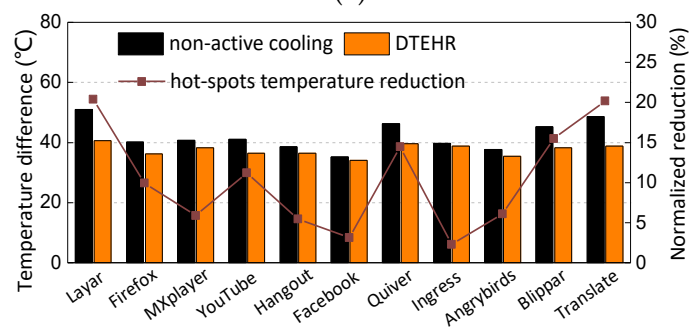

(c)

Figure 10 Temperature and the corresponding temperature reduction of hot-spots under baseline 2 and DTEHR. (a) the back cover, (b) the internal components, (c) the front cover.

additional layer occupies half of the existing air block between PCB and rear case. Thus, no additional thickness is needed for DTEHR. Moreover, the additional weight is $2 \mathrm{~g}$, which is acceptable.

\subsection{Evaluation Results for DTEHR}

The temperature distribution when the smartphone is operating with cellular-only is similar to when it is operating with Wi-Fi. Therefore, we compare DTEHR with baselines based on the simulation results with $\mathrm{Wi}-\mathrm{Fi}$ in this Section.

DTEHR can efficiently reduce temperatures of the internal hot-spots by an average of $12.8^{\circ} \mathrm{C}$. Fig. 9 shows 


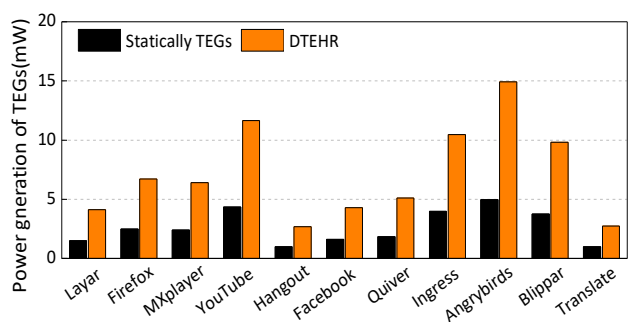

Figure 11 Comparison of power generation of TEGs between the baseline 1 and our proposed DTEHR across the benchmarks.

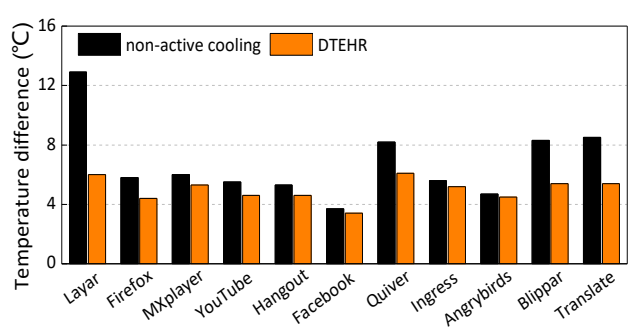

(a)

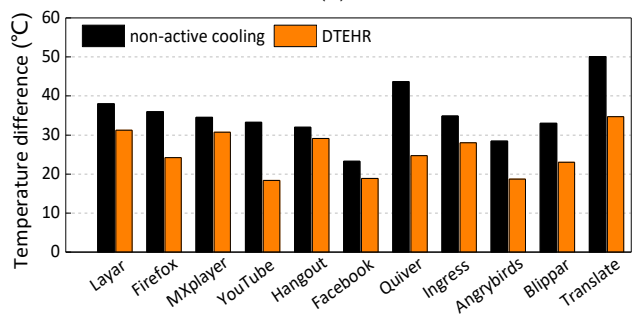

(b)

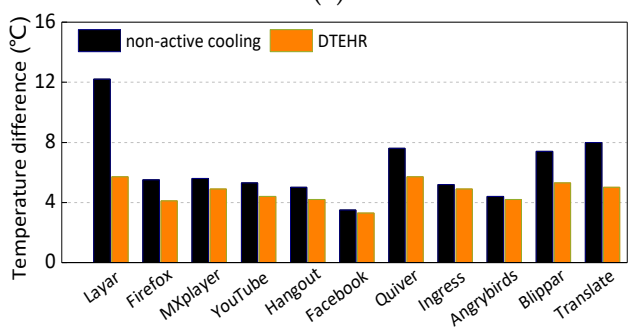

(c)

Figure 12 Temperature difference between the hot-spots and cold area under baseline 2 and DTEHR. (a) the back cover, (b) the internal components, (c) the front cover.

the amounts of TECs power consumption and the corresponding internal hot-spots' temperature reductions across the benchmarks chosen in this paper. It shows that the cooling power cost by each app is around $29 \mu \mathrm{W}$, while temperature reductions of the internal hot-spots range from $4.4^{\circ} \mathrm{C}$ to $23.8^{\circ} \mathrm{C}$. The different reductions are caused by their different cooling time, based on our cooling policy.

Fig. 10 shows the comparison of the hot-spots' temperatures between baseline 2 and DTEHR. The hotspots' temperatures are shown in the left y-axis and the reductions normalized to baseline 2 are shown in the right y-axis. Fig. 10 (b) shows temperatures of the internal hotspots. Significant hot-spots' temperatures reductions are achieved with our DTEHR, which keep internal hot-spots

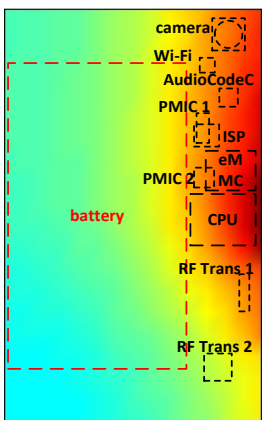

(a)

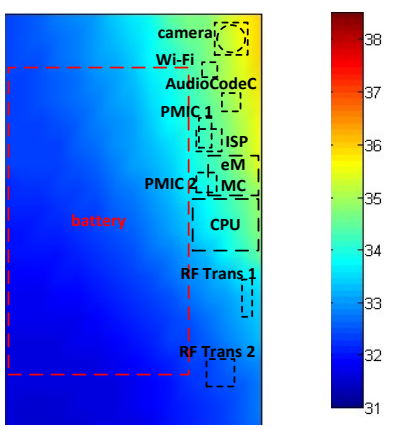

(b)
Figure 13 Temperature distributions on the back cover with Angrybirds (a) under the baseline 2, (b) under DTEHR.

under $70^{\circ} \mathrm{C}$ to avoids chip lifespan. Figs. 10 (a) and (c) show the hot-spots' temperatures of the back and the front surfaces while running various apps under baseline 2 and DTEHR. The maximum surface temperature remaining below $41^{\circ} \mathrm{C}$ under DTEHR, which improves the user experience.

Fig. 11 shows the different power generations when running various applications under DTEHR and baseline 1. It shows that power generated by DTEHR is three times that of static TEGs across the benchmarks, and the generated power is more than hundreds of times that TECs cost. The extra energy then is stored in MSCs battery to extend the usage of the smartphone.

Fig. 12 (b) shows the temperature differences between the internal hot-spots and cold areas under baseline 2 and DTEHR. In the non-active cooling design, the differences between hot-spots and the cold areas are from $23.3^{\circ} \mathrm{C}$ (Facebook) up to $50.1^{\circ} \mathrm{C}$ (Google Translate). Our dynamic TEGs absorb heat from hot areas and release to the cold areas to balance the smartphone temperature. The result shows DTEHR reduces the internal temperature differences by an average of $9.6^{\circ} \mathrm{C}$. Since temperatures of internal components directly affects temperatures of the smartphone surface, the decrease of the internal temperature difference leads to a smaller surface temperature difference. Figs. 12 (a) and (c) show the temperature difference between the surface hot-spots and cold areas under baseline 2 and our proposed DTEHR. It illustrates that the surface temperature difference stays below $6^{\circ} \mathrm{C}$ under DTEHR design across the chosen apps.

Fig. 13 illustrates the temperature maps on the back cover while running Angrybirds under baseline 2 and DTEHR. It shows that the temperature of the back cover stays below $37^{\circ} \mathrm{C}$ with DTEHR, which significantly improve the user experience.

Overall, the maximum temperature in both surface covers and internal components under DTEHR is significantly lower than that of baseline 2. Moreover, the temperature difference under DTEHR is reduced to an acceptable level. Comparing with baseline 1 of statically TEG-based hot-spots' cooling, DTEHR generates much more power. 


\section{Related Work}

Our work focuses on the thermal issue of over-heating and massive temperature difference on the smartphone surface, which significantly affects the user experience. Research areas in this study include dynamic TEGs, hotspots management, and the reusing with the MSCs battery. In this Section, we describe prior work in these domains and compare them to DTEHR.

Electricity generator with TEGs: A promising research on small-scale TEGs has been conducted recently. As TEGs becomes flexible and printable, there are some prior studies utilizing TEGs for body heat harvesting on wearable smart devices [39-42]. Moreover, nanomaterials have been used to optimize TEG material with nanotechnologies for TEG fabrication [43-48]. Traditional TEGs are mounted statically on the generator device, can only transfer heat from one side of the device to another side. Different from prior works, our work proposed (1) a dynamic TEGs module, and (2) the option to transfer internal heat from a hot area to cold components. Our dynamic TEGs can not only transfer heat from chip to ambient air but also from one hot component to one cold component. Thus, our dynamic TEGs can efficiently balance the smartphone temperature.

Hot-spots management with TECs: As more and more performance-intensive applications are running on smartphones, hot-spots management has been a critical part of smartphone design. Current smartphones often use DVFS to control the chip temperature of the smartphone to avoid hot-spots.

With the increase of smartphone performance requirements, passive cooling, including thermal pads $[49,50][32]$ and phase-change material (PCM) [51-53] have been proposed to slow down the heat transmission process and avoid hot-spots. Recently, an active cooling method with an active cooling fan [9] has been applied to the smartphone. However, this active cooling method significantly affects user experience due to its huge space requirement. Compared with above methods, our TECbased active cooling is a feasible solution to avoid high temperature because it's negligible size and weight $[37,38]$ $[54,55]$. It focuses on hot-spots and the cooling power is supplied from our dynamic TEGs.

MSCs-based energy reusing: As an emerging technology, MSCs have been integrated with active microsystems [16] [21] [56]. Wu et al. [21] demonstrated graphene-based in-plane interdigital MSCs, leading to a better result in area capacitance and stack capacitance. Kyeremateng et al. [16] showed how the threedimensional architecture could be developed to improve MSCs energy-storage capacity. We build our reusing system with MSCs to store the power generated from our dynamic TEGs for extending the battery life.

\section{Conclusions}

This paper proposes DTEHR, a mobile thermal energy harvesting reusing framework that exploits dynamic TEGs to generate power by transferring heat from hot areas to cold components. When running power-intensive applications, DTEHR powers TECs for hot-spots cooling to avoid surface hot-spots. What is more, an MSCs battery is integrated for storing extra-generated power from TEGs.

We further use MPPTAT to characterize the thermal dissipation of DTEHR. Evaluation results show that: (1) DTEHR ensures the surface temperature staying below the human tolerance limitation. Moreover, DTEHR avoids internal smartphone chip damage as the internal hotspots' temperatures remain below $70^{\circ} \mathrm{C}$. (2) DTEHR reduces the temperature difference between different components, which improves the user experience. (3) Power generated from DTEHR can not only be used for supplying hot-spots cooling but also be used for recharging the MSCs battery. To sum up, we believe that DTEHR provides a feasible hardware solution for future smartphone power and thermal management.

\section{Acknowledgments}

We thank all the anonymous reviewers for the invaluable and insightful comments to make this paper better. This work is supported in part by NSF grants 1527535, 1423090, 1320100, 1117261, 0937869, 0916384, 0845721 (CAREER), 0834288, 0811611, 0720476, by SRC grants 2008-HJ-1798, 2007-RJ-1651G, by Microsoft Research Trustworthy Computing, Safe and Scalable Multicore Computing Awards, and by three IBM Faculty Awards.

\section{References}

[1] "How Pokemon Go affects your phone's battery life and data CNET." [Online]. Available: https://www.cnet.com/howto/pokemon-go-battery-test-data-usage/. [Accessed: 11-Aug-2017].

[2] "Pokemon GO Drains A LOT MORE Battery since the last update!" [Online]. Available: http://pokemongohub.net/pokemon-go-drainsa-lot-more-battery-since-the-last-update/. [Accessed: 29-Mar-2017].

[3] "Pokemon GO Overheating Phones, Causing App to Freeze." [Online]. Available: http://www.gosunoob.com/pokemongo/overheating-phones-causing-app-freeze/. [Accessed: 11-Aug2017].

[4] D. Brooks and M. Martonosi, "Dynamic thermal management for high-performance microprocessors," Proceedings HPCA Seventh International Symposium on High-Performance Computer Architecture, Monterrey, 2001, pp. 171-182.

[5] K. Skadron, M. R. Stan, K. Sankaranarayanan, W. Huang, S. Velusamy and D. Tarjan, "Temperature-aware microarchitecture," 30th Annual International Symposium on Computer Architecture, 2003. Proceedings. 2003, pp. 2-13.

[6] F. Paterna, J. Zanotelli, and T. S. Rosing. "Ambient variation-tolerant and inter components aware thermal management for mobile system on chips," In Proceedings of the Conference on Design, Automation \& Test in Europe, DATE '2014, pp. 1-6.

[7] M. Song, Y. Hu, H. Chen and T. Li, "Towards Pervasive and User Satisfactory CNN across GPU Microarchitectures," 2017 IEEE International Symposium on High Performance Computer 
Architecture (HPCA), Austin, TX, 2017, pp. 1-12.

[8] "Backup battery," 1996. [Online]. Available: https://en.wikipedia.org/wiki/Backup_battery. [Accessed: 06-Aug2017].

[9] R. Grimes, E. Walsh, and P. Walsh, "Active cooling of a mobile phone handset," Applied Thermal Engineering, Volume 30, Issue 16, 2010, pp. 2363-2369.

[10] F.L. Tan, C.P. Tso, "Cooling of mobile electronic devices using phase change materials," Applied Thermal Engineering, Volume 24, Issues 2-3, 2004, pp. 159-169.

[11] H. Chen, Y. Dai, H. Meng, Y. Chen, and T. Li, "Understanding the characteristics of Mobile Augmented Reality Applications" 2018 IEEE International Symposium on Performance Analysis of Systems and Software.

[12] V. Wienert, H. Sick, and J. Z. Mühlen, "Local thermal stress tolerance of human skin," Anasth. Intensivther. Notfallmed, vol. 18, no. 2, 1983, p. 88-90.

[13] S. K. Roy, "An equation for estimating the maximum allowable surface temperatures of electronic equipment," in 2011 27th Annual IEEE Semiconductor Thermal Measurement and Management Symposium, 2011, pp. 54-62.

[14] "Thermoelectric_generator" [Online]. Available: https://en.wikipedia.org/wiki/Thermoelectric_generator. [Accessed: 07-Aug-2017].

[15] "Thermoelectric_cooling” [Online]. Available: https://en.wikipedia.org/wiki/Thermoelectric_cooling. [Accessed: 07-Aug-2017].

[16] N. A. Kyeremateng, T. Brousse, and D. Pech, "Microsupercapacitors as miniaturized energy-storage components for on-chip electronics," Nature Nanotechnology, vol. 12, no. 1, 2017, pp. 7-15.

[17] B. E. Conway, "Electrochemical Supercapacitors: Scientific Fundamentals and Technological Applications," Kluwer, 1999.

[18] "SuperCapacitor.”: https://en.wikipedia.org/wiki/Supercapacitor.

[19] C. Shen, S. Xu, Y. Xie, M. Sanghadasa, X. Wang and L. Lin, "A Review of On-Chip Micro Supercapacitors for Integrated SelfPowering Systems," in Journal of Microelectromechanical Systems, vol. 26, no. 5, pp. 949-965

[20] "Stretchy supercapacitors power wearable electronics - American Chemical Society." [Online]. Available: https://www.acs.org/content/acs/en/pressroom/newsreleases/2016/a ugust/stretchy-supercapacitors-power-wearable-electronics.html. [Accessed: 07-Aug-2017].

[21] Z. Wu, K. Parvez, X. Feng, and K. Müllen, "Graphene-Based in-Plane Micro-Supercapacitors with High Power and Energy Densities." Nature Communications 4 (2013): 2487. PMC. Web. 21 Jan. 2018.

[22] "Fourier's Law," A-to-Z Guide to Thermodynamics, Heat and Mass Transfer, and Fluids Engineering. [Online]. Available: http://www.thermopedia.com/content/781/. [Accessed: 07-Aug2017].

[23] "Ftrace." [Online]. Available: https://www.kernel.org/doc/Documentation/trace/ftrace.txt. [Accessed: 07-Aug-2017].

[24] W. Huang, M. R. Stan, K. Skadron, K. Sankaranarayanan, S. Ghosh, and S. Velusam. "Compact thermal modeling for temperature-aware design," in Proceedings of the 41st annual Design Automation Conference (DAC '04). 2004, ACM, New York, NY, USA, pp. 878-883.

[25] F. Aquilante, L. Boman, J. Boström, H. Koch, R. Lindh, A. S. Merás, and T. B. Pedersen, "Cholesky decomposition techniques in electronic structure theory," in Linear-Scaling Techniques in Computational Chemistry and Physics, Springer, 2011, pp. 301-343.

[26] M. Computing, "DAQ-USB-2408," 2014. [Online]. Available: http://www.mccdaq.com/PDFs/manuals/USB-2408-Series.pdf. [Accessed: 07-Aug-2017].

[27] “Layar.” [Online]. Available: https://www.layar.com/. [Accessed: 29Mar-2017].

[28] "Quiver." [Online]. Available: http://www.quivervision.com/. [Accessed: 29-Mar-2017].

[29] "Ingress." [Online]. Available: https://www.ingress.com/. [Accessed: 07-Aug-2017].

[30] "Blippar.” [Online]. Available: https://blippar.com/en/. [Accessed: 29-Mar-2017].

[31] "Google Translate." [Online]. Available: https://play.google.com/store/apps/details?id=com.google.android.ap ps.translate. [Accessed: 29-Mar-2017].

[32] S. P. Gurrum, D. R. Edwards, T. M.-Golder, J. Akiyama, S. Yokoya, J. F. Drouard, and F. Dahan, "Generic thermal analysis for phone and tablet systems," 2012 IEEE 62nd Electronic Components and Technology Conference, San Diego, CA, 2012, pp. 1488-1492.

[33] Q. Xie, J. Kim, Y. Wang, D. Shin, N. Chang and M. Pedram, "Dynamic thermal management in mobile devices considering the thermal coupling between battery and application processor," 2013 IEEE/ACM International Conference on Computer-Aided Design (ICCAD), San Jose, CA, 2013, pp. 242-247

[34] Q. Xie, M. J. Dousti and M. Pedram, "Therminator: A thermal simulator for smartphones producing accurate chip and skin temperature maps," 2014 IEEE/ACM International Symposium on Low Power Electronics and Design (ISLPED), La Jolla, CA, 2014, pp. 117-122.

[35] "Common Material Properties - Thermoelectric." [Online]. Available: https://thermal.ferrotec.com/technology/thermoelectric-referenceguide/thermalrefab/. [Accessed: 07-Aug-2017].

[36] D. Park, S. Park, K jeong, H Jeong, J. Y. Song, and M. Cho, "Thermal and Electrical Conduction of Single-Crystal Bi2Te3 Nanostructures Grown Using a One Step Process.” Scientific Reports 6 (2016): 19132. PMC. Web. 21 Jan. 2018.

[37] S. Biswas, M. Tiwari, T. Sherwood, L. Theogarajan and F. T. Chong, "Fighting fire with fire: Modeling the datacenter-scale effects of targeted superlattice thermal management," 2011 38th Annual International Symposium on Computer Architecture (ISCA), San Jose, CA, 2011, pp. 331-340.

[38] S. Lee, D. Pandiyan, Jae-sun Seo, P. E. Phelan and C. J. Wu, "Thermoelectric-based sustainable self-cooling for fine-grained processor hot spots," 2016 15th IEEE Intersociety Conference on Thermal and Thermomechanical Phenomena in Electronic Systems (ITherm), Las Vegas, NV, 2016, pp. 847-856.

[39] V. Leonov, P. Fiorini, and R. J. M. Vullers, "Theory and simulation of a thermally matched micromachined thermopile in a wearable energy harvester," Microelectronics Journal, Volume 42, Issue 4, 2011, pp. 579-584.

[40] S. J. Kim, J. H. We, and B. J. Cho, "A wearable thermoelectric generator fabricated on a glass fabric," Energy Environ. Sci., vol. 7, no. 6, pp. 1959-1965, 2014.

[41] Z. Cao, E. Koukharenko, M.J. Tudor, R.N. Torah, and S.P. Beeby, "Flexible screen printed thermoelectric generator with enhanced processes and materials," Sensors and Actuators A: Physical, vol 238, 2016, pp. 196-206.

[42] Q. Wu and J. Hu, "A novel design for a wearable thermoelectric generator based on 3D fabric structure," Smart Materials and Structures, vol. 26, no. 4

[43] A. J. Minnich, M. S. Dresselhaus, Z. F. Ren, and G. Chen, "Bulk nanostructured thermoelectric materials: current research and future prospects," Energy Environ. Sci., vol. 2, no. 5, 2009, pp. 466-479.

[44] B. Poudel, Q. Hao, Y. Ma, Y. Lan, A. Minnich, B. Yu, X.Yan, D.Wang, A. Muto, D. Vashaee, X. Chen, J. Liu, M. S. Dresselhaus, G. Chen, and Z. Ren, "High-Thermoelectric Performance of Nanostructured Bismuth Antimony Telluride Bulk Alloys," Science, vol. 320, Issue 5876, 2008, pp. 634-638.

[45] R. Hu, B. A. Cola, N. Haram, J. N. Barisci, S. Lee, S. Stoughton, G. Wallace, C. Too, M. Thomas, A. Gestos, M. E. Cruz, J. P. Ferraris, A. A. Zakhidov, and R. H. Baughman, "Harvesting Waste Thermal Energy Using a Carbon-Nanotube-Based Thermo-Electrochemical Cell," Nano Letters, vol. 10, no. 3, Mar. 2010, pp. 838-846.

[46] G. Delaizir, J. Monnier, M. Soulier, R. Grodzki, B. Villeroy, J. Testard, J. Simon, C. Navone, C. Godart, "A new generation of high performance large-scale and flexible thermo-generators based on $(\mathrm{Bi}, \mathrm{Sb}) 2(\mathrm{Te}, \mathrm{Se}) 3$ nano-powders using the Spark Plasma Sintering technique," Sensors and Actuators A: Physical, vol. 174, 2012, pp. $115-122$.

[47] Y. Yang, Z. Lin, T. Hou, F. Zhang, and Z. L. Wang, "Nanowirecomposite based flexible thermoelectric nanogenerators and selfpowered temperature sensors," Nano Research, vol. 5, Issues 12, 2012, pp. 888-895.

[48] K. Zhang, J. Qiu, and S. Wang, "Thermoelectric properties of PEDOT nanowire/PEDOT hybrids," Nanoscale, vol. 8, no. 15, 2016, pp. 8033- 
8041.

[49] Z. Luo, H. Cho, X. Luo, and K. Cho, "System thermal analysis for mobile phone," Applied Thermal Engineering, vol. 28, no. 14-15, 2008, pp. 1889-1895.

[50] J. Rajmond and A. Fodor, "Thermal management of embedded devices," Proceedings of the 36th International Spring Seminar on Electronics Technology, Alba Iulia, 2013, pp. 30-34.

[51] M. Skach, M. Arora, C. Hsu, Q. Li, D. Tullsen, L. Tang, and J. Mars, "Thermal time shifting: Leveraging phase change materials to reduce cooling costs in warehouse-scale computers," in ISCA '15

Proceedings of the 42nd Annual International Symposium on Computer Architecture, 2015, pp. 439-449.

[52] G. Setoh, F.L. Tan, and S.C. Fok, "Experimental studies on the use of a phase change material for cooling mobile phones," International Communications in Heat and Mass Transfer, Volume 37, Issue 9, 2010, pp 1403-1410.
[53] Y. Tomizawa, K. Sasaki, A. Kuroda, R. Takeda, and Y. Kaito, "Experimental and numerical study on phase change material (PCM) for thermal management of mobile devices," Applied Thermal Engineering, vol. 98, 2016, pp. 320-329.

[54] K. Fukutani and A. Shakouri, "Design of Bulk Thermoelectric Modules for Integrated Circuit Thermal Management," in IEEE Transactions on Components and Packaging Technologies, vol. 29, no. 4, 2006, pp. 750-757.

[55] C.A. Gould, N.Y.A. Shammas, S. Grainger, and I. Taylor, "Thermoelectric cooling of microelectronic circuits and waste heat electrical power generation in a desktop personal computer," Materials Science and Engineering: B, vol 176, no 4, 2011, pp. 316325.

[56] M. F. El-Kady and R. B. Kaner, "Scalable fabrication of high-power graphene micro-supercapacitors for flexible and on-chip energy storage," Nature Communication 2013;4:1475/1-1475 